EPJ manuscript No.

(will be inserted by the editor)

\title{
Haloes and Clustering in Light, Neutron-Rich Nuclei
}

\author{
NA Orr ${ }^{1}$ \\ Laboratoire de Physique Corpusculaire, IN2P3-CNRS, ISMRa et Université de Caen, F-14050 Caen cedex, France
}

November 15, 2018

\begin{abstract}
Clustering is a relatively widespread phenomena which takes on many guises across the nuclear landscape. Selected topics concerning the study of halo systems and clustering in light, neutron-rich nuclei are discussed here through illustrative examples taken from the Be isotopic chain.
\end{abstract}

PACS. 27.20.+n $6 \leq A \leq 19-21.45 .+\mathrm{v}$ Few-body systems

\section{Introduction}

Until relatively recently cluster studies have been confined to the region encompassing the line of beta stability where the rôle of $\alpha$-clustering has long been established [1]. As ' clustering is expected to manifest itself most strongly near thresholds [2], exotic structures might be expected to form in the more weakly bound systems found near the neutron ' and proton driplines. In practical terms the exploration of clustering in nuclei far from stability has become techni' cally feasible over the last 15 years through the advent of radioactive beams and associated techniques. This is demonstrated most clearly by the discovery and subsequent probing of the nuclear halo.

In the present paper selected topics concerning the study of halo and molecular states in light, neutron-rich nuclei which have undergone developments since the previous conference in this series are discussed. Following a brief review of work on $x \alpha$ :Xn molecular states, emphasis is placed on new techniques for probing correlations in two-neutron halo nuclei. Very recent work exploring the means to produce and detect multineutron clusters is also presented. As they exhibit many of the facets of clustering and structural evolution far from stability the examples discussed here have been selected from the neutron-rich Be isotopes.

\section{Nuclear Molecular Clusters}

It has long been established that the $\alpha$-particle plays an important rôle in the structure of light $\alpha$-conjugate $(\mathrm{A}=4 \mathrm{n})$ nuclei [1]. This is a direct consequence of the strongly bound character of the ${ }^{4} \mathrm{He}$ nucleus and the weakness of the $\alpha-\alpha$ interaction, as evidenced by the unbound nature of ${ }^{8} \mathrm{Be}$. Whilst an excess of neutrons (or protons) may nä̈vely be expected to dilute any underlying $\alpha$-cluster structures, theoretical [3, 4 and recent experimental work
[5.6] indicate that molecular type structures such as $\alpha$ chains "bound" by valence nucleons may also occur. The persistence of such cluster structures in systems lying away from the line of beta-stability is well illustrated, as will be discussed here, by the beryllium isotopes, for which the $\alpha$ - $\alpha$ system may be regarded as the basis.

From a theoretical point of view, prescriptions such as the Molecular-Orbital Model (MO) [3] or the Two-Centre Shell Model [7], in which valence nucleons are added to the single-particle orbits arising from the two-centre potential, provide a successful and conceptually appealing framework within which to describe the properties of these nuclei. Moreover these orbits may be viewed as the analogues of the $\sigma$ and $\pi$-orbitals associated with the covalent binding of atomic molecules. The development of fully fledged Antisymmeterised Molecular Dynamics calculations (AMD), as discussed by Horiuchi in his contribution to this conference, is of particular interest as the A-nucleon system is modelled without any a priori imposition of an underlying cluster structure. Recent calculations, in particular, suggest the existence of two-centred structures in the $\mathrm{Be}, \mathrm{B}$ and $\mathrm{C}$ isotopic chains with valence neutron density distributions exhibiting the features of molecular orbitals [4.

From an experimental perspective, von Oertzen [5] has compiled systematic evidence for the existence of dimers in ${ }^{9-11} \mathrm{Be}$ and ${ }^{9-11} \mathrm{~B}$. In the case of ${ }^{9} \mathrm{Be}$, for example, the presence of a valence neutron results in a bound (Borromean) system, the ground and excited states of which may be understood in terms of a three-body $\alpha: n: \alpha$ molecular structure. In particular, the rotational bands based on the ground and low-lying states exhibit large deformations consistent with the associated molecular configurations.

In the case of ${ }^{10} \mathrm{Be}$, the experimental evidence for molecular configurations is rather less well documented. Beyond the established $0_{2}^{+}, 2_{2}^{+}$and $1_{1}^{-}-4_{1}^{-}$states, the locations of the $\mathrm{J}=5$ [5] and 6 members of the of the negative parity band, as well as the $\mathrm{J}=4$ and 6 members of the positive parity band have been postulated following 


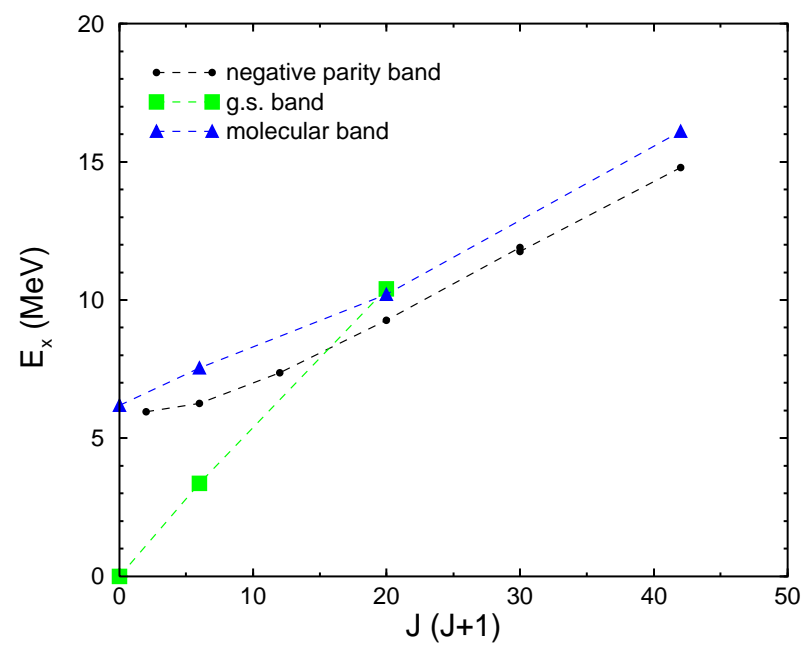

Fig. 1. Spin-energy systematics for states observed in ${ }^{10} \mathrm{Be}$ (from 91). The trajectories for the postulated positive and negative parity molecular bands are indicated.

recent studies of the $\alpha^{-}{ }^{6} \mathrm{He}$ breakup of ${ }^{10} \mathrm{Be}^{*}[8,9]$. As displayed in Fig. 1 [9], the spin-energy trajectories for the bands based on the $0_{2}^{+}$and $1_{1}^{-}$states at $\sim 6 \mathrm{MeV}$ are consistent with large deformations as expected for molecularlike $\alpha: 2 \mathrm{n}: \alpha$ structures. Moreover, the location of the bandheads just below the threshold for $\alpha+{ }^{6} \mathrm{He}$ decay is in accordance with the considerations of Ikeda describing the formation of clusters [2]. Theoretical support for the postulated molecular states may be found in AMD calculations, whereby well developed $\alpha: 2 \mathrm{n}: \alpha$ configurations are predicted for the $0_{2}^{+}$and $1_{1}^{-}$bands 佃. Coupled-channel calculations presented in these proceedings by Ito and collaborators also provide support for the molecular nature of the band based on the $0_{2}^{+}$. Furthermore, extended MO model calculations 11] have demonstrated the that the $\mathrm{O}_{2}^{+}$state may be well characterised by valence neutrons in occupying the $\sigma$-orbital.

Given the existence of such molecular-type structures in ${ }^{10} \mathrm{Be}$, the question naturally arises as to the existence of similar structures in more neutron-rich systems. In this context the dripline nucleus ${ }^{12} \mathrm{Be}$ has been investigated via inelastic scattering at $35 \mathrm{MeV} /$ nucleon. Evidence was found in this measurement for the breakup into ${ }^{6} \mathrm{He}+{ }^{6} \mathrm{He}$ and $\alpha+{ }^{8} \mathrm{He}$ of states $(\mathrm{J}=4,6,8)$ in the excitation energy range $10-20 \mathrm{MeV}$ which exhibited spin-energy systematics characteristic of a rotational band [6]. Moreover the inferred momenta of inertia $\left(\hbar^{2} / 2 \Im=0.15 \pm 0.04 \mathrm{MeV}\right)$ and bandhead energy $(10.8 \pm 1.8 \mathrm{MeV})$ of the observed states are consistent with the cluster decay of a molecular structure which may be associated with $\alpha: 4 \mathrm{n}: \alpha$ configurations. As reported at this conference by Saito et al., an experiment undertaken very recently at RIKEN using two neutron removal from an energetic ${ }^{14}$ Be beam has uncovered evidence for possible $0^{+}$and $2^{+}$states some 1.7 and $2.7 \mathrm{MeV}$ above the ${ }^{6} \mathrm{He}+{ }^{6} \mathrm{He}$ breakup threshold. Interestingly these states conform well to the spin-energy systematics established in our original study.
Somewhat indirect evidence for the existence of the states observed in the two breakup experiments may be found in the observation of highly excited states in the ${ }^{9} \mathrm{Be}\left({ }^{15} \mathrm{~N},{ }^{12} \mathrm{~N}\right){ }^{12} \mathrm{Be}$ reaction which are consistent with the spin-energy systematics of a deformed rotational band [10]. The theoretical investigation of molecular configurations in ${ }^{12} \mathrm{Be}$ represents a somewhat more challenging venture than the lighter mass Be isotopes. Nevertheless, efforts are being made, as evidenced by the contribution to this conference by Ito and coworkers and recent papers by Itagaki et al. [11] and Descouvemont and Baye [12].

As suggested by von Oertzen [5] and more recently by Itagaki et al 113, the neutron-rich $\mathrm{C}$ isotopes may be expected to exhibit $3 \alpha: X n$ cluster structures. In this context, we have attempted to observe such states in the ${ }^{12} \mathrm{C}\left({ }^{16} \mathrm{C},{ }^{16} \mathrm{C}^{*} \rightarrow{ }^{10,12} \mathrm{Be}+{ }^{6,4} \mathrm{He}\right)$ reaction at $35 \mathrm{MeV} /$ nucleon [14. Careful analysis of the fragment coincidences could, however, only put an upper limit of some $30 \mu \mathrm{b}$ on the yield to states in these decay channels. The inability to access such states by inelastic scattering may arise from a much smaller overlap between the ${ }^{16} \mathrm{C}$ ground state and the cluster states than in the case of ${ }^{12} \mathrm{Be}$. In this context transfer reactions using projectiles $\left({ }^{6,8} \mathrm{He}\right)$ and targets $\left({ }^{6} \mathrm{Li},{ }^{9} \mathrm{Be},{ }^{12} \mathrm{C}\right)$ exhibiting cluster structure may provide a more efficient means of accessing molecular states in neutron-rich nuclei.

Beyond the extension of cluster studies to more exotic systems, one of the most pressing issues is the determination of partial decay widths. Clearly this is not a trivial task, as evidenced by the difficulties encountered in the study of clustering in stable nuclei. In the case the light, neutron-rich nuclei discussed here, the presence of relatively few decay channels and the more favourable signalto-background ratio when employing radioactive beams may, however, facilitate such measurements.

\section{Correlations in Two-Neutron Halo Nuclei}

Clustering also appears in the guise of neutron haloes in ground states near the neutron drip-line. Arguably the most intriguing are the Borromean two-neutron halo nuclei $\left({ }^{6} \mathrm{He},{ }^{11} \mathrm{Li}\right.$ and $\left.{ }^{14} \mathrm{Be}\right)$, in which the two-body subsystems are unbound. Such behaviour naturally gives rise to the question of the correlations between the constituents. Even in the case of the most studied of these nuclei, ${ }^{6} \mathrm{He}$ and ${ }^{11} \mathrm{Li}$, little is known in this respect. Over the last three years we have developed a number of techniques for exploring the correlations within two-neutron halo systems [15, 16.

We have explored the spatial configuration of the halo neutrons at breakup through application of the technique of intensity interferometry - an approach first developed for stellar interferometry by Hanbury-Brown and Twiss in Australia in the 1950's and 60's [17 and later extended to source size measurements in high energy collisions [18]. The principle behind the technique is as follows: when identical partiles are emitted in close proximity in spacetime, the wave function of relative motion is modified by the FSI and quantum statistical symmetries [19] — in the 
case of halo neutrons the overwhelming effect is that of the FSI 15]. Intensity interferometry relates this modification to the space-time separation of the particles at emission as a function of the four-momenta of the particles through the correlation function $C_{\mathrm{nn}}$, which is defined as,

$$
C_{\mathrm{nn}}\left(p_{1}, p_{2}\right)=\frac{d^{2} n / d p_{1} d p_{2}}{\left(d n / d p_{1}\right)\left(d n / d p_{2}\right)}
$$

where the numerator is the measured two-particle distribution and the denominator the product of the independent single-particle distributions 15. As is generally the case, the single-particle distribution have been generated in our work via event mixing. Importantly, in the case of halo neutrons special consideration must be given to the strong residual correlations [15]. Experimentally care needs to be taken to eliminate cross talk [20].

As a first step, kinematically complete measurements of breakup on a $\mathrm{Pb}$ target of ${ }^{6} \mathrm{He},{ }^{11} \mathrm{Li}$ and ${ }^{14} \mathrm{Be}$ were analysed 15]. The choice of a high- $Z$ target was made to privilege Coulomb induced breakup, whereby the halo neutrons may in a first approximation act as spectators and for which simultaneous emission may be expected to occur. The correlation functions derived from the data, assuming simultaneous emission, were compared to an analytical formalism based on a Gaussian source [21]. Neutron-neutron separations of $r_{n n}^{R M S}=5.9 \pm 1.2 \mathrm{fm}$ $\left({ }^{6} \mathrm{He}\right), 6.6 \pm 1.5 \mathrm{fm}\left({ }^{11} \mathrm{Li}\right)$ and $5.6 \pm 1.0 \mathrm{fm}\left({ }^{14} \mathrm{Be}\right)$ were thus extracted. These results appear to preclude any strong dineutron component in the halo wavefunctions at breakup. It is interesting in this context to compare these results to the RMS neutron-proton separation of $3.8 \mathrm{fm}$ in the deuteron (the only bound two nucleon system).

The same analysis has been applied to dissociation of ${ }^{14} \mathrm{Be}$ by a $\mathrm{C}$ target, in order to investigate the influence of the reaction mechanism. A result which hints at a somewhat larger separation, $r_{n n}^{r m s}=7.6 \pm 1.7 \mathrm{fm}$, was obtained. This raises the question as to whether simultaneous emission can be assumed a priori. In principle, the analysis of the correlation function in two dimensions, transverse and parallel to the total momentum of the pair, would allow for the unfolding of the source size and lifetime 21]. Such an analysis requires a much larger data set than presently available. The two-neutron halo, however, is far less complex than the systems usually studied via interferometry (for example, heavy-ion collisions 19). Moreover, the simple three-body nature of the system breaking up suggests that any delay in the emission of one of the neutrons will arise from core-n FSI/resonances in the exit channel, a process that may be expected to be enhanced for nuclear induced breakup.

Correlations in three-particle decays are commonly encountered in particle physics and are typically analysed using plots of the squared invariant masses of particle pairs $\left(M_{i j}^{2}, M_{i k}^{2}\right)$, with $M_{i j}^{2}=\left(p_{i}+p_{j}\right)^{2}$; a technique developed by the Australian physicist Richard Dalitz in the early 1950's [23]. In these Dalitz plot representations, FSI or resonances lead to a non-uniform population of the surface within the kinematic boundary defined by energymomentum conservation and the decay energy. In the

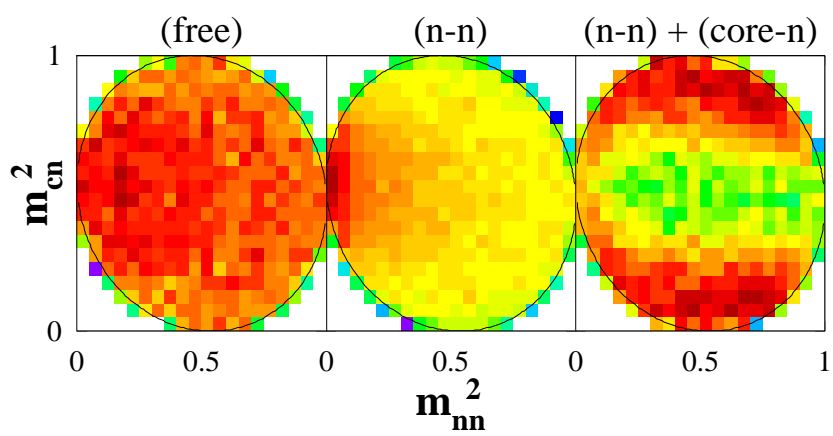

Fig. 2. Dalitz plot for the simulated decay (see text) of ${ }^{14} \mathrm{Be}$. In the left panel no FSI are included.

present case, the core $+\mathrm{n}+\mathrm{n}$ system exhibits a distribution of decay energies $\left(E_{\mathrm{d}}\right)$. The $E_{\mathrm{d}}$ associated with each event will thus lead to a different kinematic boundary, and the resulting plot containing all events cannot be easily interpreted. We have thus introduced a normalised invariant mass,

$$
m_{i j}^{2}=\frac{M_{i j}^{2}-\left(m_{i}+m_{j}\right)^{2}}{\left(m_{i}+m_{j}+E_{\mathrm{d}}\right)^{2}-\left(m_{i}+m_{j}\right)^{2}}
$$

which ranges between 0 and 1 (that is, a relative energy $E_{i j}=M_{i j}-m_{i}-m_{j}$ between 0 and $E_{\mathrm{d}}$ ) for all events and exhibits a single kinematic boundary. Examples of how n$\mathrm{n}$ and core-n FSI may manifest themselves in the Dalitz plot for the decay of ${ }^{14} \mathrm{Be}$ are illustrated in Fig. 2, whereby events have been simulated according to the simple interacting phase-space model described in ref. 16. The inputs were an $E_{\mathrm{d}}$ distribution following that measured [22], the $C_{\mathrm{nn}}$ obtained with the $\mathrm{C}$ target, and a core-n resonances with $\Gamma=0.3 \mathrm{MeV}$ at $E_{0}=0.8 \mathrm{MeV}$. Note that due to the normalisation the (squared) core-neutron invariant mass does not present a simple structure directly related to the energy of the resonance/FSI [16].

As described in ref. [16], the data from the dissociation of ${ }^{14} \mathrm{Be}$ on $\mathrm{C}$ and $\mathrm{Pb}$ targets have been analysed in terms of Dalitz plots and compared to the interacting phase-space calculations. The results for breakup on the $\mathrm{Pb}$ target are shown in Fig 3. The good agreement obtained through the inclusion of only the n-n FSI suggests that, within the presently available statistics, the two-neutrons are emitted essentially simultaneously.

Despite much reduced statistics for the triple coincidence events needed for this analysis, breakup on the $\mathrm{C}$ target is consistent with a finite delay between the emission of the two neutrons [16]. This may be related to the population of core-neutron resonances as suggested by more classical analyses of ${ }^{12}$ Be-neutron events [24]. By fixing the n-n source size to that derived from the $C_{n n}$ for the $\mathrm{Pb}$ target, an average delay in the emission for breakup by $\mathrm{C}$ of $150_{-150}^{+250} \mathrm{fm} / c$ was estimated.

Finally, in the context of the influence of the reaction mechanism, it is worthwhile noting that whilst some $35 \%$ of the two-neutron removal cross section on the $\mathrm{Pb}$ target is attributable to nuclear induced breakup [22], the 


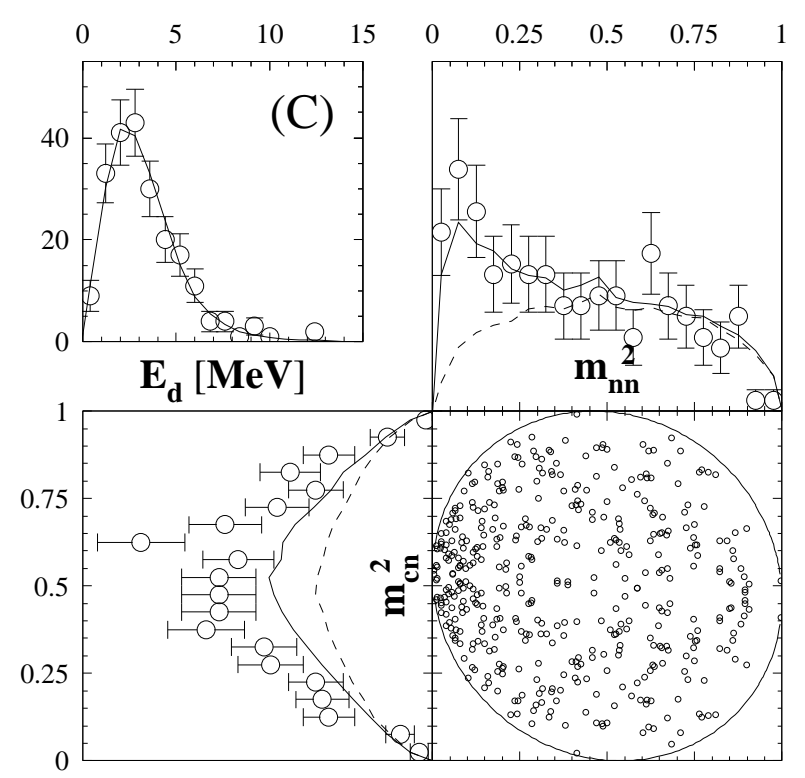

Fig. 3. Dalitz plot and the projections onto the squared invariant masses for the dissociation of ${ }^{14} \mathrm{Be}$ by $\mathrm{Pb}$. The lines are the phase-space model simulations with/without (solid/dashed) n$n$ FSI. The inset shows the measured $E_{d}$ spectrum.

requirement of two neutrons in coincidence with the ${ }^{12} \mathrm{Be}$ core in the present analysis reduces this to some $15 \%$ - approximately half of the two-neutron removal cross section arises from absorption.

\section{Multineutron Clusters}

It is interesting to speculate that multineutron halo nuclei and other very neutron-rich systems may contain components of the wavefunction in which the neutrons present a relatively compact cluster-like configuration. If this were to be the case, then the dissociation of beams of such nuclei may offer a means to produce bound multineutron clusters (if they exist) and, more generally study multineutron correlations.

To date the majority of searches for multineutron systems have relied on very low (typically $\sim 1 \mathrm{nb}$ ) cross section double-pion charge exchange $(\mathrm{D} \pi \mathrm{CX})$ and heavyion transfer reactions (see, for example, refs [25, 26]). In the case of dissociation of an energetic beam of a very neutron-rich nucleus, relatively high cross sections (typically $\sim 100 \mathrm{mb}$ ) are encountered. Thus, even only a small component of the wavefunction corresponding to a multineutron cluster could result in a measurable yield with a moderate secondary beam intensity. Furthermore the backgrounds encountered in $\mathrm{D} \pi \mathrm{CX}$ and heavy-ion transfer reactions are obviated in direct breakup.

The difficulty in this approach lies in the direct detection of a ${ }^{A} \mathrm{n}$ cluster. One avenue we are exploring is to use the detection of the recoiling proton in a liquid scintillator [27]. The advantage of a liquid scintallator is that neutrons may be discriminated from the $\gamma$ and cosmic-ray

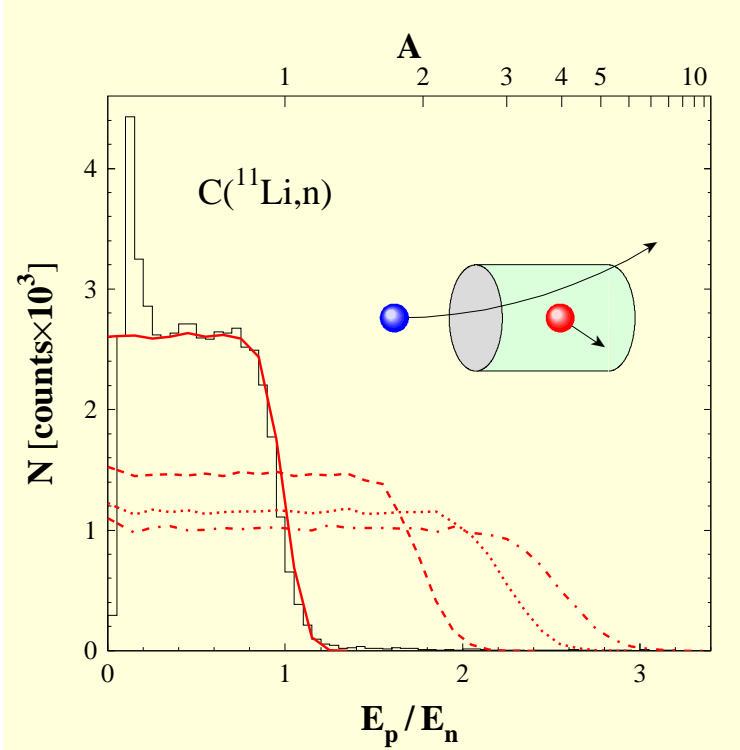

Fig. 4. $E_{p} / E_{n}$ for $\mathrm{A}=1$ (solid line), 2 (dashed), 3 (dotted) and 4 (dot-dashed). In the case of $\mathrm{A}=1$, comparison is made to single neutron events from ${ }^{11} \mathrm{Li}+\mathrm{C}$.

backgrounds. Careful source and cosmic-ray calibrations permit the charge deposited and hence the energy $\left(E_{p}\right)$ of the recoiling proton to be determined. This may be compared to the energy derived from the measured timeof-flight $\left(E_{n}\right)$ : for a single neutron and an ideal detector, $E_{p} / E_{n} \leq 1$; for a realistic detector with finite resolution the limit is $\sim 1.4$. In the case of a multineutron cluster $\left({ }^{A} \mathrm{n}\right)$ $E_{p}$ can exceed the incident energy per nucleon and $E_{p} / E_{n}$ will take on a range of values extending beyond $1.2-$ up to $\sim 3$ in the case of $\mathrm{A}=4$ (Fig. 4).

Analyses of data already available from the breakup of ${ }^{14} \mathrm{Be}$ by $\mathrm{C}$ show some 7 events with $E_{p} / E_{n}$ i. 1.4 (Fig. 5 ). Furthermore all 7 events are in coincidence with ${ }^{10} \mathrm{Be}$ fragments. Intriguingly, preliminary estimates suggest that the detection of two or more neutrons in a single module (the most likely source of background) should produce much less that one event with $E_{p} / E_{n i 1} 1.4$ in coincidence with ${ }^{10} \mathrm{Be}$. Further detailed analysis of these events and all possible sources of background is presently underway. In addition, higher statistics measurements employing beams such as ${ }^{8} \mathrm{He}$ are currently being planned.

It is a pleasure to thank the members of the E281 and E295 collaborations and, in particular, the DEMON and CHARISSA crews. I would also like to draw attention to the key rôles played by Martin Freer (Be cluster studies), Miguel Marqués (correlations and neutron clusters) and Marc Labiche ( ${ }^{14}$ Be breakup). Finally, the support provided by the staffs of LPC and GANIL in preparing and executing the experiments is gratefully acknowledged.

This work was funded under the auspices of the IN2P3CNRS (France) and EPSRC (United Kingdom). Additional support from the ALLIANCE programme (Ministère des Affaires Etrangères and British Council) and the Human Capital 


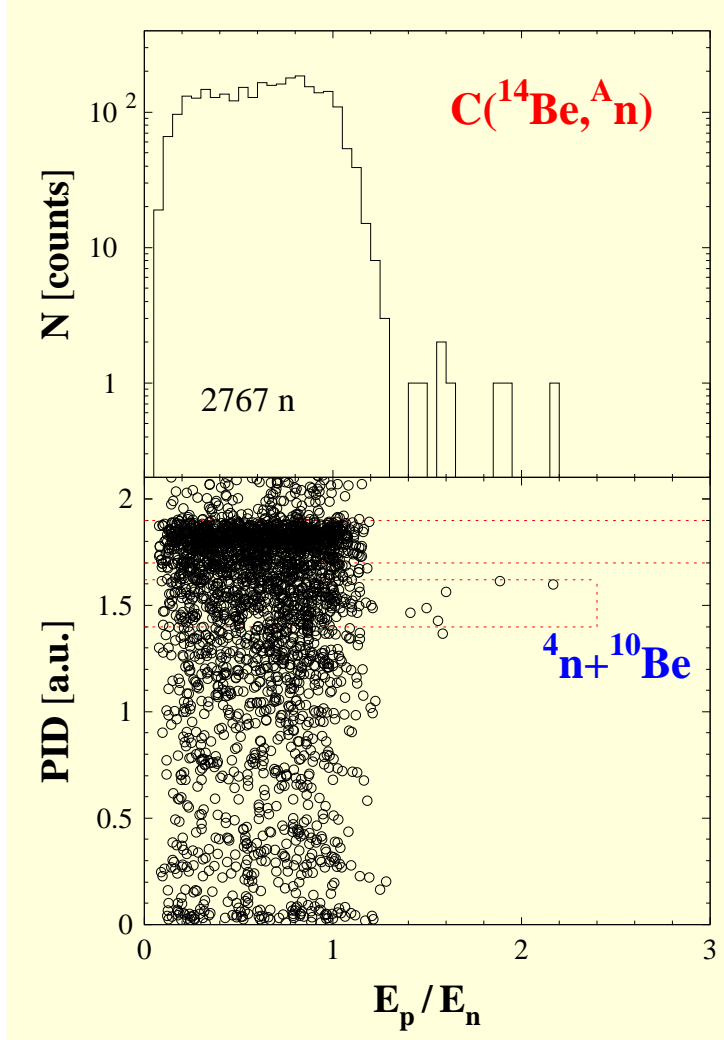

Fig. 5. Particle identification (PID) versus $E_{p} / E_{n}$ for the breakup of ${ }^{14} \mathrm{Be}$ by $\mathrm{C}$. The two horizontal bands deliniated by the dotted lines correspond to the ${ }^{12} \mathrm{Be}$ (upper) and ${ }^{10} \mathrm{Be}$ (lower) fragments.

and Mobility Programme of the European Community (Access to Large Scale Facilities) is also acknowledged.

\section{References}

1. M. Freer, A. Merchant, J. Phys. G23 (1997) 261

2. K. Ikeda, Prog. Theor. Phys. (Japan) Suppl. (1968) 464

3. M. Seya et al., Prog. Theor. Phys. (Japan) 65 (1968) 205

4. Y. Kanada-En'yo et al, Phys. Rev. C60 (1999) 064304 and refs therein

5. W. von Oertzen, Z. Phys. A354 (1996) 37; A357 (1997) 355

6. M. Freer et al., Phys. Rev. Lett. 82 (1999) 1383

7. J.M. Eisenberg, W. Greiner, Nuclear Theory (North Holland, Amsterdam, 1975)

8. N. Soíc et al., Europhys. Lett. 34 (1996) 7

9. M. Freer et al., Phys. Rev. C62 (2001) 034301

10. H.G. Bohen et al., AIP Conf. Proc. 495 (1999) 303

11. N. Itagaki et al., Phys. Rev. C62 (2000) 034301

12. P. Descouvemont, D. Baye, Phys. Lett. B505 (2001) 71

13. N. Itagaki et al., RIKEN-AF-NP-386 (2001)

14. P.J. Leask et al., J. Phys. G (in press)

15. F.M. Marqués et al., Phys. Lett. B476 (2000) 219

16. F.M. Marqués et al., nucl-ex/0101004; Phys. Rev. C (in press)

17. R. Hanbury-Brown, R. Twiss, Philos. Mag. 45 (1954) 663
18. G. Goldhaber et al., Phys. Rev. 120 (1960) 300

19. D.H. Boal et al., Rev. Mod. Phys. 62 (1990) 553

20. F.M. Marqués et al., Nucl. Inst. Meth. A450 (2000) 109

21. R. Lednicky, L. Lyuboshits, Sov. J. Nucl. Phys. 35 (1982) 770

22. M. Labiche et al., Phys. Rev. Lett. 86 (2001) 600

23. R.H. Dalitz, Philos. Mag. 44 (1953) 1068

24. K.L. Jones, Thesis, University of Surrey (2000); N.A. Orr, nucl-ex/0011002 and refs therein

25. J. Grüter et al., Eur. Phys. J. 4 (1999) 5

26. H.G. Bohlen et al., Nucl. Phys. A583 (1995) 775

27. F.M. Marqués et al., in preparation 